\title{
Central-line-associated bloodstream infections in a resource-limited South African neonatal intensive care unit
}

\author{
C Geldenhuys, ${ }^{1}$ MB ChB, FCPaed (SA), MMed (Paed), DCH; A Dramowski, ${ }^{1}$ MB ChB, FCPaed (SA), MMed (Paed), Cert ID, DCH; \\ A Jenkins, ${ }^{2}$ B Nursing, Dipl Paed Nursing, A Bekker, ${ }^{1} \mathrm{MB}$ ChB, FCPaed (SA), MMed (Paed), Cert Neonatology \\ ${ }^{1}$ Department of Paediatrics and Child Health, Faculty of Medicine and Health Sciences, Stellenbosch University, Cape Town, South Africa \\ ${ }^{2}$ Neonatal Intensive Care Unit, Tygerberg Children's Hospital, Cape Town, South Africa
}

Corresponding author: C Geldenhuys (chandre.geldenhuys@yahoo.com)

\begin{abstract}
Background. The rate of central-line-associated bloodstream infection (CLABSI) in South African (SA) public sector neonatal intensive care units (NICUs) is unknown. Tygerberg Children's Hospital (TCH), Cape Town, introduced a neonatal CLABSI surveillance and prevention programme in August 2012.

Objectives. To describe CLABSI events and identify risk factors for development of CLABSI in a resource-limited NICU.

Methods. A retrospective case-control study was conducted using prospectively collected NICU CLABSI events matched to four randomly selected controls, sampled from the NICU registry between 9 August 2012 and 31 July 2014. Clinical data and laboratory records were reviewed to identify possible risk factors, using stepwise forward logistic regression analysis.

Results. A total of 706 central lines were inserted in 530 neonates during the study period. Nineteen CLABSI events were identified, with a CLABSI rate of 5.9/1 000 line days. CLABSI patients were of lower gestational age ( $28 \mathrm{v}$. 34 weeks; $p=0.003)$, lower median birth weight (1 $170 \mathrm{~g} \mathrm{v} .1975 \mathrm{~g} ; p=0.014$ ), had longer catheter dwell times ( $>4$ days) (odds ratio (OR) 5.1 (95\% confidence interval (CI) 1.0 - 25.4); $p=0.04$ ) and were more likely to have had surgery during their NICU stay (OR 3.5 (95\% CI $1.26-10)$; $p=0.01$ ). Significant risk factors for CLABSI were length of stay $>30$ days (OR 20.7 (95\% CI $2.1-203.2) ; p=0.009)$ and central-line insertion in the operating theatre (OR 8.1 (95\% CI 1.2 - 54.7); $p=0.03)$. Gram-negative pathogens predominated (12/22; 54\%), with most isolates (10/12; $83 \%)$ exhibiting multidrug resistance.

Conclusion. The TCH NICU CLABSI rate is similar to that reported from resource-limited settings, but exceeds that of high-income countries. Prolonged NICU stay and central-line insertion in the operating theatre were important risk factors for CLABSI development. Intensified neonatal staff training regarding CLABSI maintenance bundle elements and hand hygiene are key to reducing CLABSI rates.
\end{abstract}

S Afr Med J 2017;107(9):758-762. DOI:10.7196/SAMJ.2017.v107i9.12124

In most low-resource countries, surveillance of healthcare-associated infection (HAI) is limited or non-existent. A meta-analysis of HAI in low- and middle-income countries (LMIC) reported infection rates double those of developed countries, and a tripling of HAI rates in intensive care units (ICUs). ${ }^{[1]}$ Central-line-associated bloodstream infections (CLABSI) are a type of device-associated HAI mainly encountered in the ICU setting. Data on CLABSI rates in LMIC are scant, particularly from neonatal intensive care unit (NICU) settings.

The use of central lines in NICUs is often unavoidable, with lines used for administration of intravenous fluids, blood products, inotropes, antibiotics and total parenteral nutrition (TPN). Even without the use of invasive devices, hospitalised neonates are at increased risk of infection owing to prematurity, poor skin integrity and prolonged hospitalisation. Resource-limited countries contributing data to the International Nosocomial Infection Control Consortium (INICC) report NICU CLABSI rates of 3 - 4-fold higher than those documented by the US National Health Surveillance System (NHSN). ${ }^{[2,3]}$

In high-income-country NICUs, CLABSI rates declined dramatically following widespread implementation of central-line bundles. ${ }^{[4]}$ A CLABSI bundle is a strategy for insertion and maintenance of central lines, which includes several evidence-based best practices implemented simultaneously. ${ }^{[5,6]}$ Central-line care bundle elements include: hand hygiene, optimal catheter-site selection, maximal barrier precautions at insertion, chlorhexidine skin antisepsis, daily review of line necessity, sterile line access, use of closed needleless intravascular catheter systems and ensuring the line dressing stays clean and intact. ${ }^{[5,6]}$

Reductions in NICU CLABSI rates have been achieved in developing countries, ${ }^{[7]}$ but data from African NICUs are extremely limited. ${ }^{[2]}$ Our study reports the first CLABSI surveillance programme data from a public sector NICU in South Africa (SA) and aims to identify risk factors for CLABSI in this setting.

\section{Methods \\ Study setting}

Tygerberg Hospital is a public sector teaching hospital in Cape Town, SA. The neonatal service provides care to both inborn babies and neonates referred from surrounding clinics and hospitals. In 2012 and 2013, 7 800 babies were born per year: 40\% were low-birthweight ( $<2500 \mathrm{~g}), 13 \%$ were very-low-birth-weight $(<1500 \mathrm{~g})$ and $6 \%$ were extremely low-birth-weight $(<1000 \mathrm{~g})$ infants. ${ }^{[8]}$ The neonatal service includes the NICU, with 8 intensive-care beds and 4 high-care beds, as well as 112 ward beds. In 2013, there were 491 admissions to the NICU and 5265 to the wards. ${ }^{[8]}$

Indications for central-line insertion in the NICU include requirement for TPN and/or inotropes, and neonates who require intravenous fluids and/or antibiotics where peripheral intravenous access is not possible or difficult to obtain. Umbilical venous catheters (UVCs) and peripherally inserted central catheters (PICCs) are first- and second-choice central lines and are inserted by paediatric registrars 
or medical officers. Central venous catheters (CVCs) and Broviac lines are inserted in patients in whom intravenous access is difficult, where attempts at insertion of other central lines have failed, and/or in post-surgical patients who need TPN. Broviac lines are inserted by the paediatric surgical team and CVCs by either the paediatric surgery or anaesthetic team.

The Tygerberg Children's Hospital (TCH) NICU CLABSI surveillance and prevention programme was implemented on 9 August 2012, with the aim of determining baseline CLABSI rates through prospective surveillance and reducing CLABSI events through the use of central-line insertion and maintenance bundles.

\section{Study design}

The prospectively compiled NICU central-line register was used to identify cases and controls. All cases within the 2-year study period (9 August 2012 - 31 July 2014) were included, with 4 randomly selected controls per CLABSI event. Research randomiser (a computer program that generates random numbers) was used to select 4 controls for each CLABSI case, matching only for the year of the programme, i.e. 9 August 2012 - 31 July 2013 or 1 August 2013 - 31 July 2014.

Hospital and laboratory records for the cases and controls were retrospectively reviewed. If a 'control' folder was not available or the information in the folder was incomplete, the next randomly selected folder number was used. Information obtained from the folders for both cases and controls included: patient demographics, details of their NICU stay, and central-line information. Additional information was obtained for all CLABSI cases: weight closest to CLABSI event onset, number of CLABSIs per patient, date of CLABSI event, pathogen isolated and antibiotic susceptibility, antibiotics used prior to and after CLABSI diagnosis, white cell count, platelet count, haemoglobin and C-reactive protein result $48-72$ hours prior to CLABSI and $24-48$ hours after CLABSI.

All positive cultures from catheter tips submitted from the NICU during the study period and all blood culture results of babies who died or were transferred to other neonatal wards with central lines in situ were evaluated for possible missed CLABSI cases. Umbilical arterial lines were excluded from this study because surveillance of these lines was not part of the programme initially and none of the CLABSI cases was attributed to them. The CLABSI register was the primary data source used to determine total patients and central-line days to calculate the CLABSI rate.

\section{Definitions}

The US Centers for Disease Control and Prevention (CDC)/ National Healthcare Safety Network (NHSN) 2014 definitions for HAI were used. ${ }^{\left[{ }^{[9]}\right.}$ CLABSI is defined as a laboratory-confirmed bloodstream infection (LC-BSI) in a patient with a central line in situ for at least 2 calendar days (where line insertion is day 1 ). It is still considered a CLABSI if an LC-BSI occurred within 1 day of line removal. The definitions for HAI and LC-BSI must be met before the definition of CLABSI can be applied, and other HAI must be excluded. The CLABSI rate per 1000 central-line days is calculated by dividing the number of CLABSIs by the number of central-line days and multiplying the result by 1000 . Line days are the total number of days of exposure to central venous catheters by all patients in the selected population and time period. Definitions of multidrug resistance applied from Magiorakos et al. ${ }^{[10]}$ were used to report on the antibiotic-susceptibility profile of pathogens. Mangram et al.' ${ }^{[11]}$ wound classification was used to classify the wounds of all the study participants who underwent surgery.

\section{Statistical analysis}

Normally distributed data were described using means and standard deviations; non-parametric data were described with medians and interquartile ranges. The $\chi^{2}$ test was used to compare demographics of CLABSI cases v. controls; $p<0.05$ was considered statistically significant. Stepwise forward logistic regression analysis was performed to identify risk factors for CLABSI, reporting odds ratios (ORs) and $95 \%$ confidence intervals (CIs).

\section{Ethical approval}

Approval of the study (including a waiver of individual informed consent) was obtained from the Health Research Ethics Committee at Stellenbosch University (ref. no. S14/07/153).

\section{Results}

Fourteen CLABSI episodes were documented in the CLABSI register. One CLABSI episode was excluded from the study because it did not meet the CLABSI definition as per CDC/NHSN 2014 guidelines. An additional six 'missed' CLABSI episodes were added following review of records from babies who died with a central line in situ and those with a positive culture from their catheter tip, yielding a total of 19 CLABSI events, for which 76 controls were selected (a total of 95 study patients). The NICU CLABSI surveillance registers documented insertion of 706 central lines into 530 neonates during the first 2 years of the programme (a total of 3187 central-line days). Nineteen CLABSI episodes in 17 patients were identified, yielding a CLABSI rate of 5.9/1 000 line days. The demographics of the study population are summarised in Table 1.

Nine of the CLABSI patients had medical indications for admission: hyaline membrane disease $(n=3)$, meconium aspiration syndrome $(n=2)$, presumed nosocomial sepsis $(n=2)$, congenital cytomegalovirus infection $(n=1)$ and pulmonary haemorrhage $(n=1)$. Eight patients who had undergone abdominal surgery during their current or previous NICU admission developed CLABSI episodes (10/19; 53\%), including one baby who had undergone surgery and who was admitted to the NICU for 107 days, experiencing three CLABSI events with different pathogens on three separate lines. Of the 8 babies who underwent surgery, 5 had a wound class III/IV (contaminated or dirty). ${ }^{[11]}$ Comparison of septic markers 48 hours prior to and at/within 24 hours after the CLABSI event confirmed the possibility of sepsis, with a decrease in white cell count, platelets and/or raised C-reactive protein. Of the 5 neonates who died (26.3\%), 3 deaths were directly attributed to the CLABSI event, 1 to necrotising enterocolitis and 1 to congenital abnormalities. The babies who died from CLABSI were of very low birth weight $(<1500 \mathrm{~g})$, premature, had medical indications for NICU admission and died within 48 hours of CLABSI diagnosis. The catheter dwell times in the ICU and time to CLABSI onset after insertion of different central-line types are summarised in Table 2.

Of the 19 CLABSI events, 16 were monomicrobial and 3 were polymicrobial, with 2 pathogens each (a total of 22 laboratoryconfirmed pathogens). Gram-negative organisms predominated $(12 / 22 ; 54 \%)$ followed by Gram-positives $(5 / 22 ; 23 \%)$ and fungi (5/22; 23\%). Antimicrobial susceptibility testing was performed on all isolates. Gram-negative organisms exhibited high rates of antimicrobial resistance: 6/7 Acinetobacter baumannii were multidrug-resistant and 4/5 Klebsiella pneumoniae produced extended-spectrum betalactamases (Table 2). Fungi isolated were Candida albicans $(n=3)$, C. parapsilosis $(n=1)$ (all fluconazole susceptible), and C. krusei $(n=1)$, susceptible to amphotericin B and voriconazole. For $14 / 19$ CLABSI events, patients were receiving antibiotics for other indications prior to the development of CLABSI. A change in the antimicrobial 
Table 1. Characteristics of the study population $(N=95)$

\begin{tabular}{|c|c|c|c|c|}
\hline Variable assessed & Patients, $n(\%)^{\star}(N=95)$ & Cases $(n=19)$ & Controls $(n=76)$ & $p$-value \\
\hline Gestational age (weeks), median (IQR) & $33(28-38)$ & $28(27-36)$ & $34(30-39)$ & 0.003 \\
\hline Gestational age premature (<37 weeks) & $66(69)$ & $15(79)$ & $51(67)$ & 0.316 \\
\hline Birth weight (g), median (IQR) & $1670(1130-2765)$ & $1170(960-2120)$ & $1975(1170-2838)$ & 0.014 \\
\hline Length of stay in NICU (days), median (IQR) & $7(4-15)$ & $26(12-83)$ & $5(4-10)$ & $<0.001$ \\
\hline \multicolumn{5}{|l|}{ Catheter dwell time in NICU (days) ${ }^{\dagger}$} \\
\hline$<4$ & $36(38)$ & $2(11)$ & $34(45)$ & \multirow[t]{3}{*}{0.007} \\
\hline $4-8$ & $39(41)$ & $9(47)$ & $30(39)$ & \\
\hline$>8$ & $20(21)$ & $8(42)$ & $12(16)$ & \\
\hline Gender (male) & $61(64)$ & $13(68)$ & $48(63)$ & 0.669 \\
\hline HIV-exposed & $21(22)$ & $5(26)$ & $16(21)$ & 0.621 \\
\hline \multicolumn{5}{|l|}{ Type of central line ${ }^{\dagger}$} \\
\hline UVC & $55(58)$ & $6(32)$ & $49(65)$ & \multirow[t]{4}{*}{0.001} \\
\hline PICC & $23(24)$ & $6(32)$ & $17(22)$ & \\
\hline CVC & $14(15)$ & $4(21)$ & $10(13)$ & \\
\hline Broviac & $3(3)$ & $3(15)$ & $0(0)$ & \\
\hline \multicolumn{5}{|l|}{ Insertion venue ${ }^{\dagger}$} \\
\hline NICU & $82(86)$ & $12(63)$ & $70(92)$ & \multirow[t]{3}{*}{0.001} \\
\hline Theatre & $8(8)$ & $6(32)$ & $2(3)$ & \\
\hline Neonatal ward & $5(5)$ & $1(5)$ & $4(5)$ & \\
\hline Respiratory support in NICU & $86(91)$ & $18(95)$ & $68(89)$ & 0.480 \\
\hline Inotropes in NICU & $43(45)$ & $11(58)$ & $32(42)$ & 0.216 \\
\hline TPN via central line & $72(76)$ & $16(84)$ & $56(74)$ & 0.338 \\
\hline Surgery during NICU stay & $28(29)$ & $10(53)$ & $18(24)$ & 0.013 \\
\hline Final outcome (survived) & $75(79)$ & $14(74)$ & $61(80)$ & 0.529 \\
\hline
\end{tabular}

treatment was made in $16 / 19$ (84\%) of patients, who showed clinical deterioration or had a change in their septic markers while awaiting blood culture results. After results of antimicrobial susceptibility testing were available, 9 patients required a change in treatment, 4 had therapy targeted to the pathogen isolated, 1 had an antimicrobial agent added, 1 remained on broad-spectrum antibiotics for suspected Gramnegative sepsis, and there was 1 for whom the notes were incomplete. Three babies died before susceptibility testing results were available.

Two-thirds of the cohort were premature, with a lower median gestational age and birth weight among the cases than the controls ( 28 v. 34 weeks; $p=0.003$, and 1170 v. $1975 \mathrm{~g} ; p=0.014$ ). The median length of stay in the NICU was 7 days, but significantly longer in neonates who developed CLABSI ( 26 v. 5 days; $p=<0.001$ ). There were a greater proportion of male infants in both groups. HIV exposure rates were similar between cases and controls; of the 5 HIVexposed neonates who developed CLABSI, all tested HIV polymerase chain reaction (PCR)-negative within the first 2 weeks of life. Seven controls were HIV-exposed, but had an unknown HIV-PCR result. The predominant line types used were UVCs (58\%) and PICCs (24\%). Broviac lines (3\%) and CVCs (15\%) were less frequently used and almost exclusively inserted in the operating theatre (all three Broviac lines and 3/4 CVCs). Infection rates of lines inserted in the NICU were significantly lower than for those inserted in the operating theatre $(12 / 78(15 \%)$ v. $7 / 15(41 \%) ; p=0.012)$. Only three Broviac lines were inserted over the 2-year study period and all three of the patients developed CLABSI.
Table 3 lists the factors associated with the development of CLABSI. In the stepwise forward logistic analysis, significant risk factors included insertion of a central line in the operating theatre (OR 8.1 (95\% CI $1.2-54.7) ; p=0.03$ ) and length of NICU stay $>30$ days (OR 20.7 (95\% CI 2.1 - 203.2); $p=0.009$ ).

\section{Discussion}

The CLABSI rate of 5.9/1 000 lines in our NICU is similar to that reported from other LMIC but much higher than CLABSI rates in high-income settings. Limited resources, understaffing, overcrowding and the high rate of premature and low-birth-weight babies probably contributed to our high CLABSI rate. In our setting, CLABSI cases occurred more commonly in babies of lower gestational age, lower birth weight, those undergoing surgical procedures and those with a catheter dwell time $>4$ days.

Patients with central lines and NICU admission for $>30$ consecutive days were 20 times more likely to develop a CLABSI event than those with shorter NICU stays. This result remained significant in multivariate analysis, although the CI was very wide. The wide CI can be attributed to the small number of cases. We postulate that the increased risk associated with CLABSI and long NICU stay may be due to increased risk of bacterial colonisation in critically ill patients with complicated and/or multisystem disease. ${ }^{[6,12]}$

In our setting, central lines inserted in the operating theatre (Broviac and CVCs) had an 8-fold increased risk of CLABSI compared with lines inserted in the NICU. The increased risk could arise 
Table 2. Characteristics of CLABSI episodes $(n=19)$

\begin{tabular}{l}
\hline Characteristics \\
Time to CLABSI after line insertion (days), \\
median (IQR)
\end{tabular}

median (IQR)

$\begin{array}{ll}\text { UVC } & 2(2-4) \\ \text { PICC } & 9(6-13) \\ \text { CVC } & 7(6-10) \\ \text { Broviac } & 20(19-35) \\ \text { Weight closest to CLABSI (g), median (IQR) } & 1400 \\ & (1029-2510)\end{array}$

Catheter dwell time in NICU (days), median (IQR)

$\begin{array}{ll}\text { All line types } & 8(14-18) \\ \text { UVC } & 4(3-5) \\ \text { PICC } & 13(8-13) \\ \text { CVC } & 8(8-11) \\ \text { Broviac } & 22(21-36)\end{array}$

Pathogens causing CLABSI $(n=22)$

Gram-positive organisms, $n$ (\%)

Coagulase-negative staphylococci $2(9)$

Enterococcus faecalis 2 (9)

Gram-negative organisms, $n(\%) \quad 12(54)$

Klebsiella pneumoniae $\quad 5(22)$

Acinetobacter baumannii $\quad 7$ (32)

Yeasts, $n(\%)$

Candida albicans $3(13)$

C. krusei $1(5)$

C. parapsilosis 1 (5)

CLABSI $=$ central-line-associated bloodstream infection; $\mathrm{IQR}=$ interquartile range $\mathrm{UVC}=$ umbilical venous catheter; PICC $=$ peripherally inserted central catheter

$\mathrm{CVC}=$ central venous catheter; $\mathrm{NICU}=$ neonatal intensive care unit

during the insertion and/or the maintenance of these lines. CLABSI events related to insertion factors usually develop within 48 - 72 hours of line insertion, suggesting that line maintenance issues were more likely responsible for Broviac and CVC CLABSIs (median time to infection onset 20 and 7 days, respectively). Insertion of Broviac lines and CVCs is also technically more difficult and often inserted in patients with difficult venous access, or where attempts at inserting other central-line types have failed. The literature has shown that TPN increases the risk of CLABSI. Broviac lines and CVCs were more likely to be used in patients who required longer periods of TPN, possibly contributing to infection risk. Another notable difference is that central-line insertion checklists are routinely completed for lines inserted in the NICU, but not for lines inserted in theatre. In light of these findings, the insertion bundle checklist should be implemented in theatre and education of all staff (not only NICU staff) involved in maintenance of surgically inserted lines undertaken. Central lines increase the risk of bloodstream infections. ${ }^{[12,13]}$ Known risk factors for CLABSI include administration of TPN, ${ }^{[12,13]}$ frequent manipulation of the line, ${ }^{[14]}$ open vascular systems, ${ }^{[15]}$ not using needleless connection $s^{[6]}$ and the use of multiple access ports. ${ }^{[6]}$ Conflicting data exist on which type of central line is associated with the highest risk for CLABSI and when prolonged catheter dwell time becomes a risk factor. ${ }^{[13,16,17]}$

In our study, nearly $80 \%$ of neonates who experienced CLABSI events were premature and half of them required surgery. Although surgery during the NICU stay and catheter dwell time in the NICU were not significant in the multivariate analysis, an association was found in the univariate analysis. The limited data available for neonates undergoing surgery suggest that premature infants, those with stomas and those with multiple surgical interventions are at highest risk of developing a CLABSI. ${ }^{[18]}$

Our study did not confirm TPN administration as a significant risk factor for CLABSI, but we were unable to compare the duration of TPN administration in cases v. controls. Surgery during NICU stay, catheter dwell time and prolonged stay in the NICU could all be risk factors related to a specific group of patients requiring longterm TPN. Future research is required to evaluate these factors and the cost-effectiveness of establishing a TPN unit for babies who are infection free but require long-term central lines for TPN.

Predominantly antibiotic-resistant Gram-negative pathogens were isolated. The high rate of antimicrobial resistance highlights the importance of our CLABSI surveillance and prevention programme and the need for continued antibiotic stewardship.

Study limitations included the small number of cases, making it difficult to extrapolate findings to the larger population; an unknown baseline CLABSI rate prior to implementing the CLABSI programme; and the retrospective collection of clinical data for the controls. This study did not specifically evaluate adherence to specific maintenance bundle elements (including number of access ports, use of needleless connectors and accessing central lines in a sterile manner). Other important CLABSI prevention strategies not explored in this study include daily assessment of the need for a central line, prevention of prematurity and promotion of early enteral feeding. Although the NICU had established a CLABSI registry, we identified six missed CLABSI events, suggesting a need for improved surveillance methods.

\section{Conclusion}

Despite these limitations, to our knowledge, this study is the first to establish CLABSI rates and risk factors in an SA public sector NICU. The establishment of a baseline CLABSI rate has assisted us with setting targets for the programme, assessing the impact of interventions, and benchmarking ourselves against other similar NICUs. LMIC are faced with unique challenges, such as staff shortages, high patient turnover, and limited resources, making prevention of HAIs, such as CLABSI, very difficult. Support from hospital management and ongoing in-service training of all staff involved in the insertion and maintenance of central lines in neonates are vital to sustain and improve our CLABSI prevention and surveillance programme. Other resource-limited NICUs can benefit from our experience in implementing a CLABSI surveillance and prevention programme.

Acknowledgements. Stellenbosch University's Faculty of Medicine and Health Sciences, the Biostatistics Unit of the Centre for Evidence-based Health Care, and the TCH NICU staff and patients.

Author contributions. CG, AB, AD, AJ: conceptualisation; $\mathrm{CG}, \mathrm{AB}, \mathrm{AD}$ : formal analysis; $C G, A J$ : investigation; $C G$ : methodology; $C G$ : writing original draft; and $\mathrm{CG}, \mathrm{AB}, \mathrm{AD}$ : writing - review and editing.

Funding. None.

Conflicts of interest. None.

1. Allegranzi B, Nejad SB, Combescure C, et al. Burden of endemic health-care-associated infection in developing countries: Systematic review and meta-analysis. Lancet 2011;377(9761):228-241. https:// doi.org/10.1016/s0140-6736(10)61458-4

2. Rosenthal VD, Maki DG, Mehta Y, et al. International Nosocomial Infection Control Consortium (INICC) report, data summary of 43 countries for 2007 - 2012. Device-associated module. Am J Infect Control 2014;42(9):942-956.

3. Dudeck MA, Edwards JR, Allen-Bridson K, et al. National healthcare safety network report, data summary for 2013, device-associated module. Am J Infect Control 2015;43(3):206-221. https://doi, org/10.1016/.ajic.2014.11.014 
Table 3. Risk factors for CLABSI

\begin{tabular}{|c|c|c|c|c|c|c|}
\hline \multirow[b]{2}{*}{ Variable } & \multicolumn{3}{|c|}{ Unadjusted analysis } & \multicolumn{3}{|c|}{ Adjusted analysis } \\
\hline & OR & 95\% CI & $p$-value & OR & 95\% CI & $p$-value \\
\hline \multicolumn{7}{|l|}{ Length of stay (days) } \\
\hline$<7$ (reference) & 1 & & & 1 & & \\
\hline $7-29$ & 3.5 & $0.9-14.4$ & 0.07 & 2.3 & $0.47-11.2$ & 0.30 \\
\hline$>30$ & 27.3 & $5.1-146.3$ & $<0.001$ & 20.7 & $2.1-203.2$ & 0.009 \\
\hline \multicolumn{7}{|l|}{ Insertion venue } \\
\hline NICU (reference) & 1 & & & & & \\
\hline Theatre & 17.5 & $3.1-97.1$ & 0.001 & 1 & $1.2-54.7$ & 0.03 \\
\hline Ward & 1.5 & $0.15-14.2$ & 0.74 & 8.12 .9 & $0.2-42.2$ & 0.437 \\
\hline \multicolumn{7}{|l|}{ Catheter dwell time (days) } \\
\hline $0-3$ (reference) & 1 & & & & & \\
\hline $4-8$ & 5.1 & $1.0-25.4$ & 0.04 & * & * & * \\
\hline$>8$ & 11.3 & $2.1-61.0$ & 0.005 & * & * & * \\
\hline \multicolumn{7}{|l|}{ Surgery during NICU stay } \\
\hline Any surgery performed & 3.5 & $1.26-10.00$ & 0.01 & * & * & * \\
\hline \multicolumn{7}{|l|}{ Birth weight (g) } \\
\hline 2500 (reference) & 1 & & & & & \\
\hline$<1000$ & 5.2 & $1.0-26.1$ & 0.04 & * & * & * \\
\hline $1000-1499$ & 3.7 & $0.9-15.8$ & 0.07 & * & * & * \\
\hline $1500-2499$ & 1.5 & $0.3-8.1$ & 0.65 & * & * & * \\
\hline
\end{tabular}

4. Shepherd EG, Kelly TJ, Vinsel JA, et al. Significant reduction of central-line associated bloodstream infections in a network of diverse neonatal nurseries. J Pediatr 2015;167(1):41-46.e3. https://doi. org/10.1016/j.jpeds.2015.03.046

5. Powers RJ, Wirtschafter DW. Decreasing central line associated bloodstream infection in neonatal intensive care. Clin Perinatol 2010;37(1):247-272. https://doi.org/10.1016/j.clp.2010.01.014

6. O'Grady NP, Alexander M, Burns LA, et al. Guidelines for the prevention of intravascular catheter-relate infections. Am J Infect Control 2011;39(4 Suppl 1):S1-S34. https://doi.org/10.1016/j.ajic.2011.01.003

7. Rosenthal VD, Dueñas L, Sobreyra-Oropeza M, et al. Findings of the International Nosocomial Infection Control Consortium (INICC), part III: Effectiveness of a multidimensional infection control approach to reduce central line-associated bloodstream infections in the neonatal intensive care units of 4 developing countries. Infect Control Hosp Epidemiol 2013;34(3):229-237. https://doi.org/10.1086/669511

8. Western Cape Government. Department of Health. Tygerberg Hospital annual report 2013. http:// www.westerncape.gov.za/dept/health/documents/annual_reports (accessed 23 April 2017).

9. Centers for Disease Control (CDC)/National Healthcare Safety Network (NHSN). National healthcare safety network overview. http://www.cdc.gov/nhsn/PDFs/pscManual/validation/pcsManual-2014-valid.pdf (accessed 23 April 2017)

10. Magiorakos AP, Srinivasan A, Carey RB, et al. Multidrug-resistant, extensively drug-resistant and pandrug-resistant bacteria: An international expert proposal for interim standard definitions for acquired resistance. Clin Microbiol Infect 2012;18(3):268-281. https://doi.org/10.1111/j.1469-0691.2011.03570.x

11. Mangram AJ, Horan TC, Pearson ML, Silver LC, Jarvis WR. Guideline for Prevention of Surgical Site Infection, 1999. Am J Infect Control 1999;27(2):97-132

12. Perlman SE, Saiman L, Larson EL. Risk factors for late-onset health care-associated bloodstream infections in patients in neonatal intensive care units. Am J Infect Control 2007;35(3):177-182. https:// doi.org/10.1016/.j.ajic.2006.01.002
13. Chien LY, Macnab Y, Aziz K, et al. Variations in central venous catheter-related infection risks among Canadian neonatal intensive care units. Pediatr Infect Dis J 2002;21(6):505-511. https://doi. org/10.1097/00006454-200206000-00006

14. Mahieu LM, de Dooy JJ, Lenaerts AE, Ieven MM, de Muynck AO. Catheter manipulations and the risk of catheter-associated bloodstream infection in neonatal intensive care unit patients. J Hosp Infect 2001;48(1):20-26. https://doi.org/10.1053/hin.2000.0930

15. Maki DG, Rosenthal VD, Salomao R, Franzetti F, Rangel-Frausto MS. Impact of switching from an ope to a closed infusion system on rates of central line-associated bloodstream infection: A meta-analysis of time-sequence cohort studies in 4 countries. Infect Control Hosp Epidemiol 2011;32(1):50-58. https:/ doi.org/10.1086/657632

16. Milstone AM, Reich NG, Advani S, et al. Catheter dwell time and CLABSIs in neonates with PICCs: A multicenter cohort study. Pediatrics 2013;132(6):e1609-e1615. https://doi.org/10.1542/peds.2013-1645

17. Yumani DFJ van den Dungen FAM, van Weissenbruch MM. Incidence and risk factors for catheterassociated bloodstream infections in neonatal intensive care. Acta Paediatr 2013;102(7):e293-e298. https://doi.org/10.1111/apa.12256

18. Klein MD, Rood K, Graham P. Central venous catheter sepsis in surgical newborns. Pediatr Surg In 2003:19(7):529-532. https://doi.org/10.1007/s00383-003-0977-6

Accepted 24 April 2017. 